\title{
Microscopy refocusing and dark-field imaging by using a simple LED array
}

\author{
Guoan Zheng, ${ }^{1, *}$ Christopher Kolner, ${ }^{1}$ and Changhuei Yang ${ }^{1,2}$ \\ ${ }^{1}$ Electrical Engineering, California Institute of Technology, Pasadena, California 91125, USA \\ ${ }^{2}$ Bioengineering, California Institute of Technology, Pasadena, California 91125, USA \\ *Corresponding author: gazheng@caltech.edu
}

Received July 14, 2011; revised September 9, 2011; accepted September 12, 2011;

posted September 13, 2011 (Doc. ID 151079); published October 5, 2011

\begin{abstract}
The condenser is one of the main components in most transmitted light compound microscopes. In this Letter, we show that such a condenser can be replaced by a programmable LED array to achieve greater imaging flexibility and functionality. Without mechanically scanning the sample or changing the microscope setup, the proposed approach can be used for dark-field imaging, bright-field imaging, microscopy sectioning, and digital refocusing. Images of a starfish embryo were acquired by using such an approach for demonstration. ( 2011 Optical Society of America OCIS codes: $110.0180,110.2945,110.3010,180.6900$.
\end{abstract}

Appropriate illumination of the specimen is an important factor in achieving high-resolution and high-quality images in microscopy and critical photomicrography. Most modern laboratory microscopes are equipped with the Köhler illumination setup, which was first introduced in 1893 by August Köhler and is now recommended by most microscope manufacturers. Such a Köhler illumination setup is composed of collector lens, field diaphragm, condenser diaphragm, and condenser lens. It can provide specimen illumination that is uniformly bright and free from glare [1]. More advanced illumination schemes have also been reported in recent years, including structured illumination [2,3], light sheet illumination [4], focusgrid illumination [5], and nondiffracted Bessel beam illumination [6].

With the maturation of LED technology, the use of LEDs as the light source for optical microscopy can bring certain cost- and usage- advantages [3,7,8]. In this Letter, we demonstrate a simple and cost-effective microscopy illumination scheme by replacing the optical condenser with a programmable LED array. The proposed illumination scheme has several advantages. 1) A conventional bright-field image can be acquired by digitally matching the illumination numerical aperture (NA) to the collection NA (i.e. NA of objective lens). 2) A dark-field image of the specimen can be acquired by simply turning on the LEDs at the edge of the array, where the illumination NA is beyond the collection NA. 3) We can sequentially turn on each individual LED and capture a sequence of specimen images. These images contain the information for different view angles and therefore we can postprocess them to digitally refocus the specimen into different depths. 4) The aforementioned imaging schemes can be accomplished simultaneously by a single LED scan process. We can select the images corresponding to LEDs within the collection NA to form a bright-field image. On the other hand, the images corresponding to LEDs beyond the collection NA can be used for dark-field imaging. Furthermore, we can realign all the images (with different view angles) to digitally refocus the specimen into different depths. 5) No mechanical moving parts are involved in the proposed scheme. The scanning rate of the LEDs can easily operate in the $\mathrm{kHz}$ domain. The limiting factor in our prototype is the capturing frame rate/data transfer rate of the camera. Because of the rapid development of the semiconductor industry, we believe that such a data transfer rate will not be a significant bottleneck for the proposed illumination scheme in the near future. 6) The proposed scheme is cost-effective and compatible with most modern laboratory microscopes.

This Letter is structured as follows. We will first describe the experimental setup of our proposed illumination scheme. Next, we will report on our results of bright-field and dark-field imaging capabilities. We will then report on our demonstration of digital refocusing of the specimen into different depths. Finally, we will draw our conclusion at the end of this Letter.

The proposed illumination scheme is shown in Fig. 1(a), where the optical condenser is replaced by an LED array. To understand the principle, we first consider only one LED lit in the LED array. The location of this LED can be denoted as $\left(x_{i}, y_{i}\right)$, as shown in Fig. 1(b). Assuming the distance (at the $z$ direction) between the LED array and specimen is ' $H$ ', the illumination NA of this LED can be defined as

$$
r / \sqrt{r^{2}+H^{2}}
$$
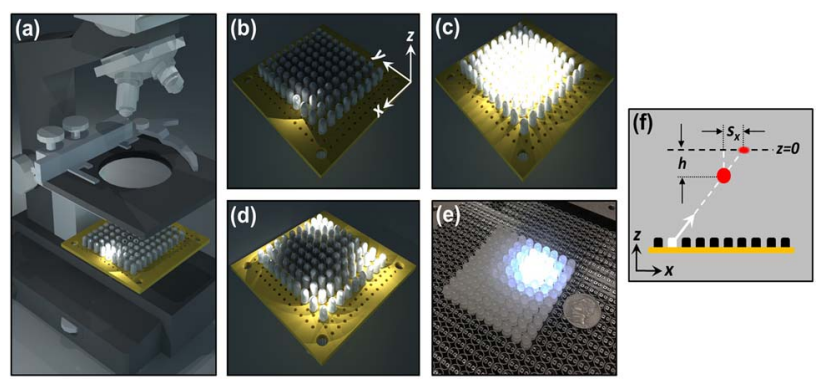

Fig. 1. (Color online) Proposed illumination scheme. (a) The optical condenser is replaced by a programmable LED array. No lens is placed between the LED array and the sample stage. (b) One LED is turned on for illumination. (c) The LEDs in central part are turned on for the bright-field imaging. (d) The LEDs at the edge are turned on for dark-field imaging. (e) The actual LED array prototype used in our experiment. (f) A simple raytrace diagram showing different $z$-planes result in different image shifts (also see Eq. (2))). 
where $r=\sqrt{\left(x_{i}-x_{c}\right)^{2}+\left(y_{i}-y_{c}\right)^{2}},\left(x_{c}, y_{c}\right)$ is the location of the LED matrix center. For microscopy brightfield imaging, the illumination NA is matched to the collection NA of the objective lens. Such a matching procedure can be performed by adjusting the size of the condenser diaphragm in the Köhler illumination setup. In the proposed scheme in Fig. 1(a), we can calculate the illumination NA for each individual LED by using Eq. (1). We can then separate them into two groups using the following criteria. Group 1: illumination NA > collection NA; group 2: illumination NA < collection NA. To achieve the bright-field illumination condition, we simply turn off the group 1 LEDs and turn on the group 2 LEDs. Figure 1(c) shows an example of NA matching by using the proposed scheme for the bright-field imaging (the LEDs at the edge, whose illumination NA is larger than the collection NA, are turned off).

Another important microscopy method is dark-field microscopy. Dark-field microscopy can be used to enhance the contrast in unstained samples [9,10]. It works by illuminating the sample with light that will not be collected by the objective lens, and thus, it will not form part of the image. In a conventional microscopy setup, a light stop is placed at the condenser to create a dark-field illumination cone. For the proposed scheme, we can simply turn on the LEDs in group 1 to collect the scattering light component from the specimen and turn off the LEDs in group 2 to reject the direct light illumination. Such an illumination scheme is illustrated in Fig. 1(d), where we need only turn on the LEDs whose illumination $\mathrm{NA}$ is larger than the collection NA.

To demonstrate the capabilities of the proposed approach, we used an Olympus BX41 microscope with a $10 \times(0.3 \mathrm{NA})$ objective lens as our demonstration imaging setup. We replaced the condenser with a programmable LED array as shown in Fig. 1(e). This LED array contained $10 * 10$ phosphor-based diffused white LEDs (Betlux BL-L513UWC, with $160^{\circ}$ wide illumination angles), which were mounted on a printed circuit board (PCB) (the PCB can support up to $25 * 25$ LEDs). The measured distance (at $z$ direction) between the sample stage and the LED array (i.e. ' $H$ ') was $80 \mathrm{~mm}$. The size of this $10 * 10$ LED array was $54 \mathrm{~mm}$ (i.e. the distance between adjacent LEDs is $6 \mathrm{~mm}$ ). This $10 * 10$ array was driven in a traditional row/column format using a Macroblock constant-current LED driver (MBI5027) and a column was selected using a demultiplexer (74HC154). An Atmel ATMEGA-328 microcontroller provided the logical control on the lines. To achieve maximum brightness, the array was driven statically rather than in normal scanning mode, eliminating the duty cycle and boosting current through the LEDs to $125 \mathrm{~mA}$ (the measured light intensity is $\sim 0.9 \mathrm{~W} / \mathrm{m}^{2}$ ) with $100 \mathrm{ohms}$ on the MBI5027 resistive input for current control.

We next used the proposed system for bright-field and dark-field imaging demonstrations (Fig. 2). We used a microscope slide of starfish embryo (Carolina Biological Supply) as the specimen. The experimental procedures can be described as follows: 1) turn on the individual LEDs one by one; 2) capture the corresponding images (total 100 frames are captured for $10 * 10$ LEDs); 3) sum the images from the group 2 LEDs to create the brightfield image, as shown in Fig. 2(a); 4) sum the images from
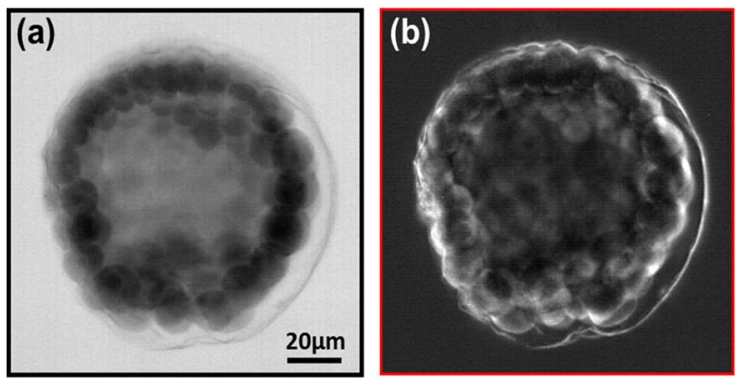

Fig. 2. (Color online) (a) Bright-field and (b) dark-field images of a starfish embryo captured using the proposed illumination scheme.

the group 1 LEDs to create the dark-field image, as shown in Fig. 2(b). The entire image capturing process required two seconds. The scanning rate of LEDs can reach the $\mathrm{kHz}$ regime and the limiting factor in our prototype was the data transfer rate of the image sensor (Aptina MT9M001; we captured images at $50 \mathrm{fps}$ ).

Next, we demonstrate a more important feature of the proposed scheme: digital refocusing. In the previous experiment shown in Fig. 2, we captured 100 frames (one for each individual LED) and then regrouped them to form a bright-field/dark-field image. In fact, this 100frame data set contained useful information on the 3D structure of the specimen and it can be used to digitally refocus the specimen into different depths.

As we can see from Fig. 1(a), each individual LED illuminated the specimen at a specific incident angle. Therefore, each frame of the captured image provided a unique perspective view of the specimen. Conceptually, the idea is similar to tilted-view microscopy [11], where the sample is mechanically tilted to provide different view angles. Based on Fourier slice theorem, each image provides us information on one slice of 3D Fourier space. Therefore, based on the captured images with different LEDs, we can reconstruct the specimen at different depths. The process is equivalent to tomography reconstruction. The data processing procedures can be described in the following steps. 1) Select the images from group 2 LEDs. 2) For different LEDs in group 2, the incident angles are different, and thus the shifts of the specimen at different depths are also different. This second step is to calculate image shifts in $x$ and $y$ directions (denoted as ' $s_{x}, s_{y}$ ') for different depths (denoted as ' $h$ ') based on the following equation:

$$
s_{x}(h)=x_{i} h / H, \quad s_{y}(h)=y_{i} h / H,
$$

where $\left(x_{i}, y_{i}\right)$ is location of the LED and ' $H$ ' is the distance between the LED array and specimen [Fig. 1(f)]. 3) Each captured image is normalized by its maximum intensity (i.e. the maximum pixel value of the image). 4) The normalized images are back shifted by the amount of $\left(s_{x}, s_{y}\right)$ and merged to one image [12]. 5) (Optional) the merged image can be further deblurred with the point spread function (PSF) of the objective lens at that certain depth ' $h$ '. The PSF can be estimated from the Airy pattern.

The result of digital refocusing following the above procedures is shown in Fig. 3(a)(1-8). Figure 3(b) is the image taken by the conventional Köhler illumination. 

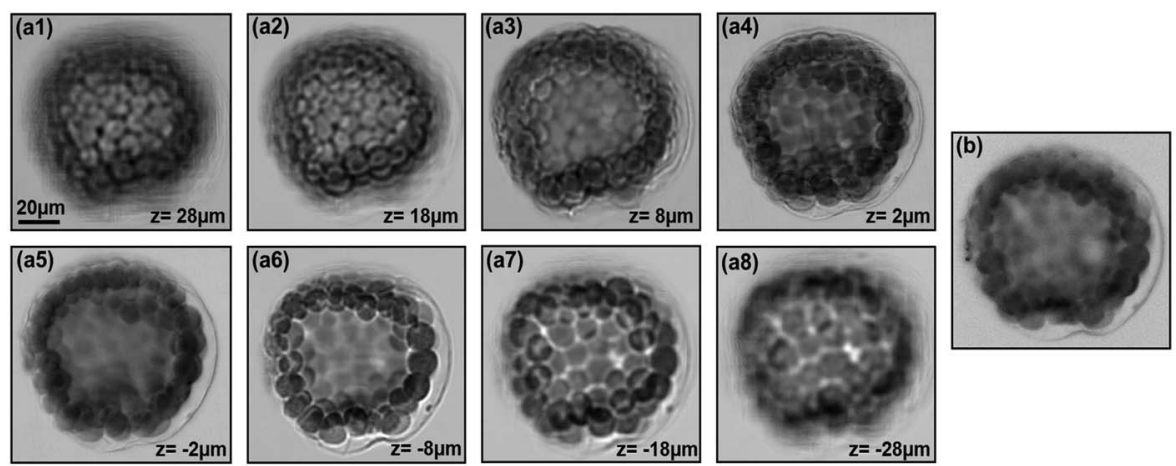

Fig. 3. (a1-a8) Based on the captured images for different individual LEDs, we can digitally refocus the starfish embryo into different depths. No mechanical scanning is involved in this process. (b) The image taken by the conventional Köhler illumination. We use a halogen lamp as the light source with an illumination NA of 0.3 (matching to $0.3 \mathrm{NA}$ objective lens).
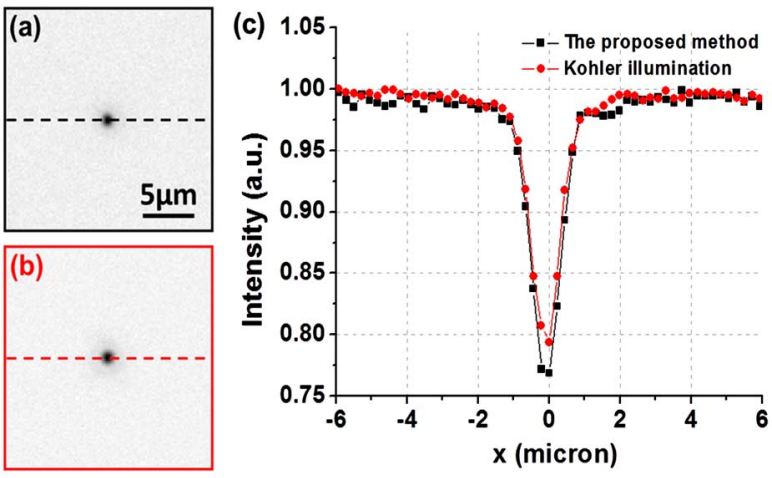

Fig. 4. (Color online) The images of a $0.5 \mu \mathrm{m}$ bead by using (a) the proposed method, (b) Köhler illumination. (c) The line traces of (a) and (b).

From this figure, we can see the cell development of the starfish embryo at different depth sections. We note that the sectioning ability of our approach is determined by the objective lens. For a conventional microscopy setup with a plane wave illumination, the captured image is a superposition of images from different depth sections through the entire depth of field. By using the proposed illumination scheme, we can separate all these different sections from the captured images. Most importantly, we can accomplish this without mechanically moving the sample or changing the setup. The image processing time was less than $0.2 \mathrm{~s}$ by using a personal computer with an Intel i7 CPU.

A special case of Eq. (2) is ' $h=0$ ', i.e. the image in focus. In this case, the reconstructed image is equivalent to the image taken by the conventional Köhler illumination approach. Figure 4 shows such a comparison. The slight difference in imäge contrast can be attributed to the difference of the light-emitting spectra of the two approaches (the point spread function of objective lens is different for different wavelengths).

To summarize, a simple microscopy illumination scheme based on a cost-effective programmable LED array is proposed in this Letter. A sequence of images is captured, one for each individual LED. Each image provides a unique view angle of the specimen. We showed that these images can be postprocessed to render a bright-field image, dark-field image, and more importantly, sectioned images at different depths. The ability to digitally refocus the specimen into different depths without mechanically scanning the sample is useful for quantitative phase imaging [13], 3D position sensing, and metrology. We note that the proposed illumination scheme is not a simple tradeoff between time and imaging functionalities. A relatively long capturing time (two seconds) allows us to acquire multiple frames, and thus the signal to noise ratio of the final result is also higher than in a single frame (Note that the bit depth of the image sensor is limited). This is similar to averaging frames to reduce noise in the conventional still microscope. We also note that the illumination angle of the diffuse LEDs we used spanned a range of 160 degrees, therefore the maximum illumination NA was close to one. To further enhance the light collecting efficiency, we can place the LED array at the black focal plane of the condenser (the rest of microscope setup would remain unchanged).

We acknowledge funding support from United States Department of Defense under grant W81XWH-09-1-0051.

\section{References}

1. J. James, Light Microscopic Techniques in Biology and Medicine (Springer, 1976).

2. M. G. L. Gustafsson, Proc. Natl. Acad. Sci. USA 102, 13081 (2005).

3. V. Poher, H. Zhang, G. Kennedy, C. Griffin, S. Oddos, E. Gu, D. Elson, M. Girkin, P. French, and M. Dawson, Opt. Express 15, 11196 (2007).

4. J. Huisken, J. Swoger, F. Del Bene, J. Wittbrodt, and E. H. K. Stelzer, Science 305, 1007 (2004).

5. J. Wu, G. Zheng, Z. Li, and C. Yang, Opt. Lett. 36, 2179 (2011).

6. F. O. Fahrbach, P. Simon, and A. Rohrbach, Nat. Photon. 4, 780 (2010).

7. V. Bormuth, J. Howard, and E. Schaffer, J. Microsc. 226, 1 (2007).

8. V. Murthy, T. F. Sato, D. F. Albeanu, E. Soucy, and M. Meister, PLoS ONE 3 (2008).

9. G. C. Cox, Optical Imaging Techniques in Cell Biology (CRC/Taylor \& Francis, Boca Raton, 2007).

10. G. Zheng, X. Cui, and C. Yang, in Proceedings of the National Academy of Sciences of India, Section A (Physical Sciences) (2010), Vol. 107, p. 9043.

11. P. J. Shaw, D. A. Agard, Y. Hiraoka, and J. W. Sedat, Biophys. J. 55, 101 (1989).

12. G. Zheng, S. A. Lee, S. Yang, and C. Yang, Lab Chip 10, 3125 (2010).

13. A. Barty, K. Nugent, D. Paganin, and A. Roberts, Opt. Lett. 23, 817 (1998). 